\title{
Betriebliche Gesundheitsförderung in Deutschland: Verbreitung und Inanspruchnahme. Ergebnisse der BIBB/ BAuA-Erwerbstätigenbefragungen 2006 und 2012
}

\author{
Workplace Health Promotion in Germany: Prevalence and Utilisation. \\ Analyses on Labour Force Surveys of the Federal Institute for Occupational \\ Safety and Health in 2006 and 2012
}

Autoren

Institute

\author{
D. Beck 1 , U. Lenhardt ${ }^{2}$
}

${ }^{1}$ Bundesanstalt für Arbeitsschutz und Arbeitsmedizin, Fachgruppe 3.5 Psychische Belastungen, Betriebliches Gesundheitsmanagement, Berlin

${ }^{2}$ Bundesanstalt für Arbeitsschutz und Arbeitsmedizin, Strategisches FuE-Management, Berlin
Schlüsselwörter

- betriebliche Gesundheitsförderung

- Verbreitung

- Inanspruchnahme

- Erwerbstätigenbefragung

Key words

workplace health promotion

- prevalence

utilization

- labour force survey

Bibliografie

Dol http://dx.doi.org/

10.1055/s-0034-1387744

Online-Publikation: 30.9.2014

Gesundheitswesen 2016;

78: 56-62

(c) Georg Thieme Verlag KG

Stuttgart · New York

ISSN 0941-3790

Korrespondenzadresse

Dr. David Beck

Bundesanstalt für Arbeitsschutz und Arbeitsmedizin

Fachgruppe 3.5 Psychische

Belastungen

Betriebliches Gesundheitsma-

nagement

Nöldnerstraße 40-42

10317 Berlin

beck.david@baua.bund.de

\section{License terms}

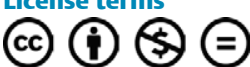

\section{Zusammenfassung}

Hintergrund und Methoden: In diesem Beitrag werden repräsentative Daten zur Verbreitung und Inanspruchnahme von Maßnahmen der Betrieblichen Gesundheitsförderung (BGF) in Deutschland dargestellt. Grundlage sind Angaben von 17870 abhängig Beschäftigten, die im Rahmen der Erwerbstätigenbefragung 2011/12 des Bundesinstituts für Berufsbildung (BIBB) und der Bundesanstalt für Arbeitsschutz und Arbeitsmedizin (BAuA) erhoben wurden. Durch Vergleich mit Daten aus der Erhebungswelle 2005/06 $(\mathrm{N}=17803)$ werden darüber hinaus mittelfristige Veränderungstendenzen im BGF-Geschehen bestimmt.

Ergebnisse: Im Jahr 2012 bestätigten 44\% der Befragten die Durchführung von BGF-Maßnahmen in ihrem Betrieb, 2006 waren es 38\%. Zuwächse zeigten sich in allen Betriebsgrößenklassen und Wirtschaftsbereichen. Beschäftigte aus kleinen Betrieben berichteten zu beiden Erhebungszeitpunkten deutlich seltener von BGFMaßnahmen als Beschäftigte aus Großbetrieben. Von den erfassten Wirtschaftsbereichen weist die Industrie die höchste Verbreitung von BGF auf, der Abstand zu den anderen Sektoren ist seit 2006 aber kleiner geworden. Der Anteil der Befragten, die im Falle durchgeführter BGF-Maßnahmen angaben, an diesen selbst teilgenommen zu haben, ging leicht zurück. Markante (und im Zeitverlauf sogar gewachsene) Unterschiede hinsichtlich der Inanspruchnahmequote bestehen vor allem zwischen klein- und großbetrieblichem Sektor.

Schlussfolgerungen: Die Ergebnisse indizieren eine moderat gestiegene Verbreitung von BGF, verweisen zugleich aber auch auf einen nach wie vor erheblichen Beratungs- und Unterstützungsbedarf insbesondere der kleinen Betriebe. Über qualitative Merkmale der hierzulande praktizierten BGF und ihre Veränderungen können auf Basis der ausgewerteten Daten allerdings keine Aussagen getroffen werden.

\section{Abstract \\ V}

Background and Methods: This article describes representative data on the availability of and individual participation in workplace health promotion (WHP) in Germany. These data are based on responses of 17870 employees which were collected within the Labour Force Survey 2011/12 of the Federal Institute for Vocational Education and Training and the Federal Institute for Occupational Safety and Health. By comparison with data from the previous wave of the survey carried out in 2005/06 ( $\mathrm{N}=17803)$, medium-term trends in WHP are identified.

Results: In 2012, 44\% of the employees reported WHP activities in their companies, as against $38 \%$ in 2006 . Increasing WHP prevalences were observed in all company sizes and economic sectors. In both surveys, employees in small companies reported significantly less WHP than those in large companies. Of all economic sectors, industrial manufacturing still showed the largest diffusion of WHP, even though the gap to other sectors has narrowed since 2006 . The proportion of respondents who individually participated in WHP (if available) has slightly declined. The most striking difference in participation rates - which has even increased over time - is between small and large companies.

Conclusions: Our findings indicate a moderately increased prevalence of WHP in Germany. At the same time, data still suggest a considerable need for WHP-related advice and support, particularly in small companies. On the basis of the analysed data, however, no statements can be made about qualitative characteristics of WHP activities in Germany. 


\section{Einleitung \\ $\nabla$}

Betriebliche Gesundheitsförderung (BGF) ist mittlerweile in den Gesundheitswissenschaften wie auch in der Sozialpolitik fest etabliert, wobei die Verständnisse von BGF allerdings deutlich variieren [1]. Die Luxemburger Deklaration von 1997 subsumiert unter BGF „alle gemeinsamen Maßnahmen von Arbeitgebern, Arbeitnehmern und Gesellschaft zur Verbesserung von Gesundheit und Wohlbefinden am Arbeitsplatz. (...) BGF ist eine moderne Unternehmensstrategie und zielt darauf ab, Krankheiten am Arbeitsplatz vorzubeugen (...), Gesundheitspotenziale zu stärken und das Wohlbefinden am Arbeitsplatz zu verbessern“ " [2]. Im deutschen Krankenversicherungsrecht ist BGF als Krankenkassenleistung bestimmt, die die Erhebung der gesundheitlichen Situation im Betrieb (einschließlich ihrer Risiken und Potenziale) umfasst, ferner die Entwicklung von Vorschlägen zur Verbesserung der gesundheitlichen Situation und zur Stärkung entsprechender Ressourcen und Fähigkeiten und schließlich auch die Unterstützung bei der Umsetzung solcher Vorschläge ( $§$ 20a Abs. 1 Sozialgesetzbuch V). Vom Arbeitsschutz hebt sich BGF insbesondere durch ihren nicht-obligatorischen Charakter sowie den auf Ressourcenstärkung zielenden Ansatz ab, sie beinhaltet dem Anspruch nach sowohl individuen- als auch strukturbezogene Maßnahmen und betrachtet die Partizipation der Beschäftigten als wesentliches Verfahrens- und Zielkriterium [1]. Die Krankenkassen als wichtigste institutionelle Träger von BGF haben ihr Engagement auf diesem Gebiet in den vergangenen Jahren deutlich verstärkt, ihre Ausgaben für BGF-Leistungen sind seit 2000 um das 3-fache auf zuletzt (2012) 46,12 Mio. Euro gestiegen [3]. Aus den jährlichen Präventionsberichten der Gesetzlichen Krankenversicherung (GKV) geht u. a. hervor, dass die Zahl der Betriebe, auf die sich diese Aktivitäten erstrecken, alleine zwischen 2010 (6473) und 2012 (8155) um gut 25\% gewachsen ist $[4,5]$.

Die in den GKV-Präventionsberichten präsentierten Daten bilden das BGF-Geschehen naturgemäß nur insoweit ab, wie Krankenkassen darin involviert sind. BGF-Aktivitäten, die ohne deren Beteiligung stattfinden (also in „Eigenregie“ der Betriebe oder mit Unterstützung anderer Instanzen, etwa der Unfallversicherungsträger [6]), werden hierdurch nicht erfasst. Ein umfassenderes Bild vom BGF-Geschehen lässt sich mittels Surveys gewinnen. Seit Mitte des vergangenen Jahrzehnts wurden Ergebnisse zur BGF aus verschiedenen Befragungsstudien veröffentlicht [7-16]. Diese haben zweifellos zu einer Verbesserung des empirischen Kenntnisstandes bezüglich BGF beigetragen, sind aber jeweils von einer oder mehreren der folgenden Limitationen gekennzeichnet:

- Beschränkung auf ausgewählte Branchen $[7,8]$ und Regionen [7-10] oder auf ein bestimmtes Betriebsgrößenspektrum $[9,11]$

- Eingeschränkte Repräsentativität der Stichprobe, etwa hinsichtlich der Betriebsgrößenstruktur [9] oder des Bildungsstandes der Befragten [12]

- Nichtberücksichtigung der Frage, in welchem Maße vorhandene BGF-Angebote von den Beschäftigten in Anspruch genommen werden [7-12,14,16]

- Beschränkung auf einen Erhebungszeitpunkt, sodass keine Trendaussagen möglich sind $[9,11-13,15,16]$

Bislang liegt also keine Studie vor, die auf Basis repräsentativer Befragungsdaten mittelfristige Veränderungen sowohl in der Verbreitung als auch in der Inanspruchnahme von BGF bundesweit und für die Gesamtheit aller Wirtschaftszweige und Be- triebgrößenklassen darzustellen vermag. Mit der nachfolgend referierten Untersuchung soll diese Lücke geschlossen werden.

\section{Datenbasis und Variablen \\ $\nabla$}

Grundlage der Untersuchung sind Querschnittsdaten aus den BIBB/BAuA-Erwerbstätigenbefragungen 2005/06 und 2011/12 des Bundesinstituts für Berufsbildung (BIBB) und der Bundesanstalt für Arbeitsschutz und Arbeitsmedizin (BAuA) (N=20000 bzw. 20036 Erwerbstätige ab 15 Jahren mit einer Arbeitszeit von mindestens $10 \mathrm{~h} /$ Woche). Die Erhebungen wurden in beiden Wellen als Telefoninterview (Computer Assisted Telephone Interview - CATI) durchgeführt [17]; nach Gewichtungen auf Basis des Mikrozensus (für Alter, Geschlecht, Familienstand, deutsch/ nicht deutsch, Stellung im Beruf, Berufsabschluss und Bundesland des Wohnorts) sind die gewonnen Daten repräsentativ für die Gesamtheit der Erwerbstätigen in Deutschland [18].

Die letzte BIBB/BAuA-Erwerbstätigenbefragung beinhaltete $c a$. 370 Fragen zu den Themenbereichen Qualifikation und Beschäftigung sowie Arbeitstätigkeiten und -bedingungen. 2005/06 und 2011/12 wurden jeweils auch 2 Fragen zur BGF gestellt: (1) „Wurden in ihrem Betrieb in den letzten 2 Jahren Maßnahmen der Gesundheitsförderung durchgeführt? (Ja, Nein, Kenne ich nicht, Weiß nicht, kA)“; (2) „Wenn ja: Haben Sie daran teilgenommen? (Ja, Nein, Weiß nicht, kA)“ [19].

Die gültigen Antworten auf diese Fragen wurden differenziert nach Betriebsgrößenklassen und Wirtschaftsbereichen, die Antworten auf die Teilnahme-Frage zusätzlich differenziert nach Alter, Geschlecht und Stellung im Beruf ausgewertet. Zur Kategorisierung der Betriebsgröße diente folgende Einteilung: bis 9 Beschäftigte (Kleinstbetriebe), 10-49 Beschäftigte (Kleinbetriebe), 50-249 Beschäftigte (mittlere Betriebe) sowie 250 und mehr Beschäftigte (Großbetriebe). Die bei der Auswertung unterschiedenen Wirtschaftsbereiche wurden in beiden Erhebungswellen direkt von den Beschäftigten erfragt, und zwar mit der Formulierung: „Wozu gehört der Betrieb, in dem Sie arbeiten? (Öffentlicher Dienst, Industrie, Handwerk, Handel, Sonstige Dienstleistungen, anderer Bereich)“.

In unsere Untersuchung wurden lediglich die Angaben von $\mathrm{Ar}$ beitern, Angestellten, Beamten und mithelfenden Familienangehörigen (nachfolgend „Beschäftigte“) einbezogen; Selbstständige, Freiberufler und freie Mitarbeiter blieben unberücksichtigt. Die Analysen basieren somit auf den Angaben von insgesamt 17803 (2006) bzw. 17870 (2012) Befragten.

Alle beschreibenden Analysen erfolgten mittels CSTABULATE der Version 18.0 von SPSS auf Basis gewichteter Daten (zur Gewichtung s.o.).

\section{Struktur der Untersuchungsstichproben}

$\nabla$

- Tab. 1 gibt einen Überblick über die Struktur der Untersuchungsstichproben. Angegeben sind sowohl die ungewichteten absoluten Häufigkeiten als auch die relativen Häufigkeiten, die wie oben beschrieben jeweils auf Basis des Mikrozensus gewichtet wurden. Für die gewichteten Prozentwerte sind zudem die jeweiligen 95\%-Konfidenzintervalle (-KI) angegeben. Im Vergleich der Untersuchungsstichproben zeigen sich Verteilungsunterschiede bei den beruflichen Statuskategorien (Erhöhung der Angestelltenquote), Altersgruppen (Anteilszuwachs der 45-Jährigen und Älteren) und Betriebsgrößenklassen (relative 
Tab. 1 Verteilung der Beschäftigten in den Analysestichproben 2005/06 und 2011/12.

\begin{tabular}{|c|c|c|c|c|c|}
\hline & & \multicolumn{2}{|c|}{$2005 / 06$} & \multicolumn{2}{|c|}{$2011 / 12$} \\
\hline & & $\mathbf{n}_{\text {roh }}$ & $\%_{\text {gew }}[95 \%-K I]$ & $\mathbf{n}_{\text {roh }}$ & $\%_{\text {gew }}[95 \%-\mathrm{KI}]$ \\
\hline Gesamt & Gesamt & 17803 & 100 & 17870 & 100 \\
\hline \multirow[t]{5}{*}{ Status } & Arbeiter & 4722 & $33[32-34]$ & 3143 & $29[28-30]$ \\
\hline & Angestellte & 11129 & $57[56-58]$ & 13090 & 64 [63-65] \\
\hline & Beamte & 1738 & $8[8-9]$ & 1501 & $7[6-7]$ \\
\hline & Mith. Familienangehörige & 191 & $1[1-2]$ & 71 & $1[1-1]$ \\
\hline & Arb./Angest. (unentschieden) & 23 & $0[0-0]$ & 65 & $0[0-1]$ \\
\hline \multirow[t]{2}{*}{ Geschlecht } & Männer & 8901 & $55[54-56]$ & 8232 & $54[53-55]$ \\
\hline & Frauen & 8902 & $45[44-46]$ & 9638 & $46[45-47]$ \\
\hline \multirow[t]{6}{*}{ Alter } & $15-24$ & 958 & $7[6-7]$ & 534 & $6[6-7]$ \\
\hline & $25-34$ & 4045 & $22[21-23]$ & 2645 & $21[21-22]$ \\
\hline & $35-44$ & 6251 & $32[31-33]$ & 4262 & $25[24-26]$ \\
\hline & $45-54$ & 4610 & $27[27-28]$ & 6294 & $31[30-32]$ \\
\hline & $55-64$ & 1812 & $11[11-12]$ & 3865 & $15[15-16]$ \\
\hline & $65-$ & 91 & $1[1-1]$ & 144 & $1[1-1]$ \\
\hline \multirow[t]{4}{*}{ Betriebsgröße } & bis 9 Beschäftigte & 2530 & $15[15-16]$ & 2126 & $12[12-13]$ \\
\hline & 10-49 Beschäftigte & 4750 & $29[28-30]$ & 4480 & $27[26-28]$ \\
\hline & 50-249 Beschäftigte & 4528 & $26[25-27]$ & 4507 & $27[26-27]$ \\
\hline & $250+$ Beschäftigte & 5289 & $30[29-31]$ & 5762 & $34[33-35]$ \\
\hline \multirow[t]{6}{*}{ Wirtschaftsbereich } & öffentlicher Dienst & 5512 & $29[28-30]$ & 5668 & $28[27-28]$ \\
\hline & Industrie & 3918 & $24[23-25]$ & 3600 & $25[24-26]$ \\
\hline & Handwerk & 1689 & $12[11-12]$ & 1593 & 12 [11-13] \\
\hline & Handel & 1894 & $12[11-12]$ & 1863 & $11[11-12]$ \\
\hline & sonstige Dienstleistungen & 3398 & $18[18-19]$ & 3351 & $18[17-19]$ \\
\hline & anderer Wirtschaftsbereich & 991 & $5[5-6]$ & 1102 & $6[6-7]$ \\
\hline
\end{tabular}

Zunahme der Großbetriebsbeschäftigten). Weitgehend unverändert geblieben sind dagegen die Verteilung auf die Wirtschaftsbereiche und die Zusammensetzung nach Geschlecht.

\section{Ergebnisse}

$\nabla$

\section{Verbreitung von BGF-Maßnahmen}

Aus 0 Tab. 2 sind - auch spezifisch für Betriebsgrößenklassen und Wirtschaftsbereiche - die ungewichteten absoluten Häufigkeiten und die gewichteten Anteile (einschl. 95\%-KI) der Befragten zu entnehmen, die die Durchführung von BGF-Maßnahmen in ihrem Betrieb in den letzten 2 Jahren bestätigten. In den Auswertungen berücksichtigt wurden nur diejenigen, die die Frage eindeutig mit Ja oder Nein beantworten konnten (jeweils gut 94\% der Befragten). Da die Wirtschaftsbereiche unterschiedliche Betriebsgrößenstrukturen aufweisen, werden für sie auch entsprechend adjustierte Anteile angegeben. Die Adjustierung erfolgte dabei auf die Betriebsgrößenklassen-Verteilung der Beschäftigten, wie sie in der gesamten Stichprobe über alle Wirtschaftsbereiche hinweg besteht.

Zwischen 2006 und 2012 hat sich der Anteil der Beschäftigten, die von BGF-Maßnahmen in ihrem Betrieb berichteten, um 6 Prozentpunkte von $38 \%$ auf $44 \%$ erhöht. Zuwächse zeigen sich in allen Betriebsgrößenklassen und Wirtschaftsbereichen, in vergleichsweise stärkerem Ausmaß aber in Großbetrieben (plus 8 Prozentpunkte) sowie im öffentlichen Dienst, wo die Häufigkeit, mit der die Durchführung von BGF bestätigt wurde, auch nach Betriebsgrößenadjustierung um 11 Prozentpunkte angestiegen ist. $\mathrm{Zu}$ beiden Erhebungszeitpunkten variierte die BGF-Häufigkeit stark in Abhängigkeit von der Betriebsgröße, in etwas geringerem Ausmaß aber auch in Abhängigkeit vom Wirtschaftsbereich. Sowohl 2006 als auch 2012 war die Quote bei Beschäftigten aus großen Betrieben fast 4-mal höher als bei Beschäftigten aus Kleinstbetrieben. Überdurchschnittlich hoch ist sie auch bei Beschäftigten aus der Industrie und dem öffentlichen Dienst selbst dann, wenn man für die Betriebsgröße adjustiert und somit rechnerisch berücksichtigt, dass in diesen Wirtschaftsbereichen der Anteil der großen Betriebe deutlich höher ist als im Handwerk oder im Handel. Letztgenannte Bereiche fallen, auch nach Betriebsgrößenadjustierung, durch vergleichsweise niedrige Anteile von Beschäftigten auf, die BGF-Maßnahmen bestätigten.

\section{Inanspruchnahme von BGF-Maßnahmen}

- Tab. 3 gibt die ungewichteten Häufigkeiten und die gewichteten Anteile (einschl. 95\%-KI) der Befragten wieder, die angaben, an BGF-Maßnahmen im eigenen Betrieb teilgenommen zu haben. In diesen Auswertungen wurden nur die Befragten berücksichtigt, die die Durchführung von BGF-Maßnahmen in ihrem Betrieb bestätigten und die Frage nach ihrer Teilnahme eindeutig mit Ja oder Nein beantworten konnten (2006: $n=6420,2012$ : $\mathrm{n}=7486$ ). Die Inanspruchnahme von BGF-Maßnahmen wird dabei auch differenziert nach Altersgruppen, Männern und Frauen, beruflichen Statusgruppen, Betriebsgrößenklassen und Wirtschaftsbereichen berichtet, im letzteren Fall auch betriebsgrößenadjustiert.

Von den Befragten, die 2006 die Durchführung von BGF-Maßnahmen in ihrem Betrieb bestätigten, gaben $65 \%$ an, daran auch teilgenommen zu haben. 2012 lag diese Quote dann 3 Prozentpunkte niedriger.

In beiden Befragungswellen war die Teilnahmequote bei Beschäftigten aus kleinen Betrieben deutlich höher als bei Beschäftigten aus großen Betrieben. Diese Diskrepanz fiel 2012 mit 18 Prozentpunkten noch größer aus als 6 Jahre zuvor, da die Teilnahmequote in Betrieben mit 1-9 Beschäftigten weniger stark zurückging als in Großbetrieben, während sie in der Größenklasse 10-49 Beschäftigte sogar anstieg. 
Tab. 2 Anteile der Beschäftigten, die die Durchführung von BGF-Maßnahmen in ihrem Betrieb bestätigten, für die Analysestichproben 2005/06 und 2011/12.

\begin{tabular}{|c|c|c|c|c|c|c|c|}
\hline & & \multicolumn{3}{|c|}{$2005 / 06$} & \multicolumn{3}{|c|}{$2011 / 12$} \\
\hline & & $\mathbf{n}_{\text {roh }}$ & $\%_{\text {gew }}[95 \%-\mathrm{KI}]$ & adj. \%* & $\mathbf{n}_{\text {roh }}$ & $\%_{\text {gew }}[95 \%-\mathrm{KI}]$ & adj. \%* \\
\hline Durchführung von BGF & ja & 6427 & 38 [37-39] & 1 & 7494 & $44[43-45]$ & 1 \\
\hline \multirow[t]{4}{*}{ Betriebsgröße } & bis 9 Beschäftigte & 357 & $15[14-17]$ & l & 343 & $18[16-20]$ & l \\
\hline & 10-49 Beschäftigte & 1142 & $26[24-28]$ & 1 & 1195 & $29[27-30]$ & 1 \\
\hline & 50-249 Beschäftigte & 1715 & $41[39-43]$ & l & 1914 & $45[43-47]$ & 1 \\
\hline & $250+$ Beschäftigte & 3032 & $59[58-61]$ & l & 3744 & 67 [65-69] & 1 \\
\hline \multirow{6}{*}{ Wirtschaftsbereich } & öffentlicher Dienst & 2117 & $41[39-43]$ & 40 & 2798 & $51[50-53]$ & 51 \\
\hline & Industrie & 2102 & $56[54-58]$ & 46 & 2058 & $60[58-63]$ & 49 \\
\hline & Handwerk & 393 & $24[22-27]$ & 29 & 395 & $28[25-31]$ & 36 \\
\hline & Handel & 418 & $23[20-25]$ & 28 & 485 & $29[26-31]$ & 35 \\
\hline & sonstige Dienstleistungen & 1022 & 31 [29-33] & 35 & 1197 & 37 [35-39] & 41 \\
\hline & anderer Wirtschaftsbereich & 291 & $32[29-36]$ & 38 & 387 & $39[35-43]$ & 42 \\
\hline
\end{tabular}

*adjustiert für Betriebsgröße

Tab. 3 Anteile der Beschäftigten, die die Teilnahme an BGF-Maßnahmen in ihrem Betrieb bestätigten, für die Analysestichproben $2005 / 06$ und $2011 / 12$.

\begin{tabular}{|c|c|c|c|c|c|c|c|}
\hline & & \multicolumn{3}{|c|}{$2005 / 06$} & \multicolumn{3}{|c|}{$2011 / 12$} \\
\hline & & $\mathbf{n}_{\text {roh }}$ & $\%$ gew $[95 \%-K I]$ & adj. \%* & $\mathbf{n}_{\text {roh }}$ & $\%$ gew $[95 \%-K I]$ & adj. \%* \\
\hline Inanspruchnahme von BGF & ja & 4101 & 65 [64-67] & I & 4527 & $62[60-63]$ & \\
\hline \multirow[t]{4}{*}{ Betriebsgröße } & bis 9 Beschäftigte & 266 & $73[66-78]$ & 1 & 246 & $71[64-77]$ & 1 \\
\hline & 10-49 Beschäftigte & 837 & 72 [69-75] & 1 & 868 & 76 [73-79] & 1 \\
\hline & 50-249 Beschäftigte & 1150 & $69[67-72]$ & 1 & 1224 & $66[63-68]$ & 1 \\
\hline & $250+$ Beschäftigte & 1725 & $59[57-61]$ & 1 & 2001 & $53[51-56]$ & 1 \\
\hline \multirow[t]{6}{*}{ Wirtschaftsbereich } & öffentlicher Dienst & 1369 & 66 [64-69] & 65 & 1668 & $60[58-62]$ & 59 \\
\hline & Industrie & 1269 & $63[60-65]$ & 66 & 1190 & $59[56-61]$ & 62 \\
\hline & Handwerk & 304 & $77[71-81]$ & 77 & 290 & 75 [69-80] & 63 \\
\hline & Handel & 261 & 65 [59-70] & 64 & 291 & $63[57-68]$ & 60 \\
\hline & sonstige Dienstleistungen & 630 & 63 [59-66] & 61 & 721 & $61[58-65]$ & 61 \\
\hline & anderer Wirtschaftsbereich & 210 & $71[64-77]$ & 70 & 246 & $67[61-73]$ & 66 \\
\hline \multirow[t]{2}{*}{ Geschlecht } & Männer & 2392 & 65 [63-67] & 1 & 2317 & $60[58-62]$ & 1 \\
\hline & Frauen & 1709 & $66[63-68]$ & 1 & 2210 & $64[62-66]$ & 1 \\
\hline \multirow[t]{5}{*}{ Alter } & $15-24$ & 189 & $59[52-66]$ & 1 & 119 & $61[53-68]$ & 1 \\
\hline & $25-34$ & 889 & 64 [61-67] & 1 & 643 & $59[56-63]$ & 1 \\
\hline & $35-44$ & 1437 & 62 [60-65] & 1 & 1093 & 62 [59-64] & 1 \\
\hline & $45-54$ & 1142 & $69[66-72]$ & 1 & 1666 & $62[60-65]$ & 1 \\
\hline & $55-64$ & 426 & $72[67-76]$ & 1 & 950 & $63[60-66]$ & 1 \\
\hline \multirow[t]{3}{*}{ Status } & Arbeiter & 1222 & $69[67-72]$ & 1 & 813 & 64 [61-67] & 1 \\
\hline & Angestellte & 2463 & $63[61-65]$ & 1 & 3232 & $60[59-62]$ & 1 \\
\hline & Beamte & 394 & $66[62-70]$ & 1 & 454 & $62[57-66]$ & 1 \\
\hline
\end{tabular}

*adjustiert für Betriebsgröße

Bei den Wirtschaftsbereichen zeigen sich dagegen nach Betriebsgrößenadjustierung kaum noch Unterschiede in den Teilnahmequoten. Eine Ausnahme bildet der deutlich erhöhte Wert im Handwerk (77\%), der allerdings nur in der Befragungswelle 2005/06 ermittelt wurde. Die betriebsgrößenadjustierten Teilnahmequoten in den verschiedenen Wirtschaftsbereichen sind 2012 fast durchgängig niedriger als 2006.

Die Teilnahmequoten zwischen Männern und Frauen sowie zwischen Arbeitern, Angestellten und Beamten unterscheiden sich nicht substanziell. In all diesen Beschäftigtengruppen ist die Inanspruchnahme von BGF-Angeboten zwischen den beiden Erhebungszeitpunkten gesunken. Zwar fiel die Gruppe der Arbeiter 2006 noch durch eine überdurchschnittliche Teilnahmequote von $69 \%$ auf. 6 Jahre später bewegte sich jedoch auch dieser Wert auf einem eher durchschnittlichen Niveau von $64 \%$.

Auch die altersgruppenspezifischen Teilnahmequoten variieren 2012 nur in geringem Maße. Das war 2006 noch anders: Damals zeigte sich noch ein Anstieg der Teilnahmequoten von 59\% bei den 15-24-jährigen bis auf 72\% bei den 55-64-jährigen Befrag- ten. Während sich die Teilnahmequote bei der jüngsten Beschäftigtengruppe im Zeitverlauf um 2 Prozentpunkte leicht erhöht hat, ist sie bei den 45-54-Jährigen um 7 und bei den 55-64-Jährigen sogar um 9 Prozentpunkte gesunken.

\section{Diskussion}

\section{Stärken und Limitationen der Untersuchung}

Eine Stärke der vorliegenden Untersuchung besteht im Umfang und in der Qualität der zugrunde liegenden Stichprobe. Das für nationale Bevölkerungssurveys außergewöhnlich große Sample zeichnet sich durch eine hohe Repräsentativität aus und ermöglicht differenzierte Auswertungen. Ein Vorteil liegt ferner darin, dass die untersuchungsrelevanten Daten zu 2 Messzeitpunkten - im Abstand von 6 Jahren - mit identischer Methodik erhoben wurden, sodass die Ergebnisse gut vergleichbar und mittelfristige Trendaussagen möglich sind. 
Die dargestellten Ergebnisse zur Verbreitung von BGF-Angeboten sind in ihrer Aussagekraft allerdings dadurch beschränkt, dass sie ausschließlich auf Wahrnehmungen der befragten Beschäftigten beruhen. Da anzunehmen ist, dass nicht alle Beschäftigten Kenntnis von den in ihrem Betrieb durchgeführten BGF-Maßnahmen erlangen, kann davon ausgegangen werden, dass ein Teil der Befragten das Vorhandensein von BGF verneinte, obwohl es sie in ihrem Betrieb tatsächlich gab oder gibt.

Ein gewichtiges Problem für die Interpretation der Erhebungsresultate ergibt sich aus der sehr einfach und allgemein gehaltenen Formulierung der beiden BGF-bezogenen Fragen, die auf eine nähere Bestimmung von BGF bzw. auf die Benennung von darunter subsumierten Instrumenten und Maßnahmen verzichtet. Für eine differenziertere Abfrage zur BGF bestand wegen des ohnehin schon sehr umfangreichen Fragenkatalogs der BIBB/ BAuA-Erhebung und des damit verbundenen Zeit- und Kostenaufwandes kein Spielraum. Infolge dessen bleibt aber unklar, auf Basis welcher Vorstellungen von BGF die Beschäftigten die ihnen hierzu vorgelegten Fragen beantworteten.

Die analysierten Daten beinhalten also keine konkreten Informationen über Art, Umfang und Dauer sowie Gegenstände und Ziele der von den Befragten bestätigten BGF-Maßnahmen. Sie lassen auch kein Urteil darüber zu, inwieweit mit diesen Maßnahmen zentralen Kriterien gesundheitsfördernder Praxis „Enabling“ und „Empowerment“ der Beschäftigten für eine gesundheitsförderliche Gestaltung ihrer Arbeit - entsprochen wird. Aussagen über das Maßnahmenprofil und die Durchführungsqualität der BGF sind auf Basis der BIBB/BAuA-Daten somit nicht möglich.

\section{Verbreitung von BGF-Maßnahmen}

Bei einem Vergleich zwischen der in der BIBB/BAuA-Befragung 2011/12 ermittelten Häufigkeit von BGF (44\%) und entsprechenden Ergebnissen aus anderen, zu ähnlichen Zeitpunkten durchgeführten Beschäftigtensurveys zeigen sich markante $A b-$ weichungen: Während in der Beschäftigtenbefragung zum DGBIndex Gute Arbeit $2012(\mathrm{~N}=4895)$ ein deutlich geringerer Anteil der Befragten (31\%) das Vorhandensein von BGF-Angeboten im eigenen Betrieb bestätigte [16], ergab die GDA-Arbeitnehmerbefragung 2011 ( $N=5$ 512) mit 58\% einen weit über dem BIBB/ BAuA-Ergebnis liegenden Wert (berechnet nach [20]). Diese Diskrepanzen dürften maßgeblich auf methodische Ursachen (insbesondere stark unterschiedliche Fragenkonstruktion) zurückzuführen sein. Für die Plausibilität des Resultats der BIBB/BAuAErhebung 2011/12 spricht, dass eine auf dieser Grundlage geschätzte betriebsbezogene BGF-Prävalenz - die ungefähr auf dem Niveau der beschäftigtenbezogenen Prävalenz im Bereich der Kleinst- und Kleinbetriebe (1-49 Mitarbeiter) liegt [15], gemäß Tab. 2 also etwa bei $26 \%$ - ziemlich exakt dem betrieblichen Verbreitungsgrad von BGF entspricht, wie er im Rahmen des bundesweiten IAB-Betriebspanels 2012 ermittelt wurde (27\%) [21].

Auch hinsichtlich des Befundes einer tendenziell gestiegenen Verbreitung von BGF stimmt unsere Untersuchung mit Ergebnissen aus den IAB-Betriebspanelbefragungen überein, die zwischen 2004 und 2012 eine Erhöhung des Anteils der Betriebe mit BGF von $20 \%$ [14] auf 27\% [21] aufzeigen. Für eine solche Tendenz sprechen auch Ergebnisse einer repräsentativen Beschäftigtenbefragung aus NRW, in der allerdings nicht nach dem Vorhandensein von BGF-Maßnahmen im Betrieb, sondern nach der Beurteilung des jeweiligen betrieblichen Engagements zur Gesundheitsförderung gefragt wurde. Hiernach war der Anteil derer, die Letzteres als „eher gut“ einstuften, zwischen 2004 und 2008 von $24 \%$ auf $36 \%$ gestiegen. Zwar hatte es in den Jahren davor einen Rückgang gegeben, der 2008 erreichte Wert lag jedoch über dem Ausgangsniveau der ersten Befragungswelle 1994 [10]. Ungeachtet der Frage nach der Qualität der Praxis belegen diese Zahlen eine insgesamt gewachsene betriebliche Akzeptanz von BGF, die ihrerseits Ausdruck jahrelanger, in vielfältigen Formen und institutionellen Zusammenhängen erfolgter Anstrengungen zur Propagierung und Verankerung dieses Ansatzes sind [22].

Ebenfalls im Einklang mit dem vorliegenden Stand der Forschung steht der hier replizierte Befund, dass BGF-Maßnahmen in kleineren Betrieben deutlich seltener umgesetzt werden als in größeren [9,11-15]. Dafür kommen eine Reihe von Erklärungen in Frage [23, 24]:

- Hemmend kann sich die kleinbetriebstypische Verdichtung von Verantwortung und Entscheidungsbefugnis in der Person des Inhabers auswirken: Dieser nimmt häufig Aufgaben im gesamten Geschäftsprozess des Unternehmens wahr und zeichnet für alle Fragen der Betriebsführung und -organisation verantwortlich. Unter diesen Umständen kann BGF auf der Liste der zu bearbeitenden Aufgaben und Probleme vergleichsweise leicht ins Hintertreffen geraten.

- Betriebe, in denen ein Betriebs-/Personalrat vorhanden ist, sind häufiger in der BGF aktiv als Betriebe ohne ein solches Gremium [14]. Allerdings gibt es nur in 6\% der Kleinbetriebe Betriebsräte [25]. Die für die BGF wichtige Initiatoren- und Promotorenfunktion der Beschäftigtenvertretungen kann hier also fast nicht wirksam werden, sodass der Unternehmer in der Regel der alleinige „Gatekeeper“ für BGF ist.

- Um eine bestimmte Anzahl von Beschäftigten mit BGF-Angeboten zu erreichen, ist im kleinbetrieblichen Bereich ein höherer Aufwand erforderlich als im großbetrieblichen. Dies stellt für Anbieter von BGF-Leistungen einen Anreiz dar, sich eher in größeren Betrieben zu engagieren. So sind die Krankenkassen nach wir vor mit fast drei Vierteln ihrer BGF-Projekte in mittleren und großen Betrieben aktiv [5].

Ein nach Wirtschaftssektoren- bzw. -branchen variierender Verbreitungsgrad der BGF wurde bereits in mehreren Befragungsstudien berichtet $[10,13,14]$. Dass diese Unterschiede nur teilweise durch die jeweiligen Betriebsgrößenstrukturen erklärbar sind, wurde bislang aber lediglich in einer einzigen Untersuchung auf Basis von Daten der BIBB/BAuA-Erwerbstätigenbefragung 2005/06 gezeigt [15]. Die im vorliegenden Artikel referierten Analysen der Daten aus 2011/12 konnten dieses Ergebnis im Wesentlichen bestätigen. Eine Besonderheit stellt allerdings der in dieser Form noch von keiner anderen Studie vorgelegte Befund dar, dass nach Kontrolle von Betriebsgrößeneffekten der öffentliche Dienst der Wirtschaftsbereich mit dem höchsten Verbreitungsgrad von BGF ist. Es kann an dieser Stelle nicht beantwortet werden, worauf die Spitzenstellung des öffentlichen Dienstes zurückzuführen ist. Mögliche Gründe sind die weniger stark durch Rentabilitätserwägungen bestimmten Arbeitsbeziehungen und die sehr viel häufiger als in der restlichen Wirtschaft anzutreffenden betrieblichen Mitbestimmungsstrukturen (Personalräte) [26].

\section{Inanspruchnahme von BGF-Maßnahmen}

Das Ziel, im Falle von BGF-Angeboten einen möglichst großen Teil der Mitarbeiter mit entsprechenden Maßnahmen zu erreichen, wird den Ergebnissen dieser Untersuchung zufolge in hohem (wenngleich seit 2006 leicht rückläufigem) Maße erreicht. 
Bei der Interpretation der ermittelten Teilnahmequote ist allerdings zu berücksichtigen, dass darin jedwede Inanspruchnahme von Angeboten innerhalb von 2 Jahren, gleich welcher Art, Dauer und Intensität, eingeht. Mit den auf deutlich abweichenden Operationalisierungen beruhenden Teilnahmequoten, wie sie in anderen Befragungsstudien ermittelt wurden $[12,13]$, ist sie nicht vergleichbar.

Zwischen Männern und Frauen zeigten sich in den BIBB/BAuAErhebungen keine sonderlich ausgeprägten Unterschiede bei der BGF-Teilnahmequote. Für ein in dieser Hinsicht bestehendes generelles Ungleichgewicht liefert die Untersuchung also keinen empirischen Anhaltspunkt. Dies schließt allerdings nicht aus, dass die Muster geschlechtsspezifischer Inanspruchnahme - wie in anderen Surveys nachgewiesen - je nach Maßnahmenart deutlich differieren können [12,13].

Auch der Zusammenhang zwischen Alter und Inanspruchnahme stellt sich den letztgenannten Studien zufolge verschieden dar, je nachdem, um welche Art von BGF-Maßnahmen es sich handelt. Während sich aber in der BIBB/BAuA-Befragung 2005/06 auch bei der allgemeinen, maßnahmenübergreifenden BGF-Teilnahmequote noch ein deutlicher Altersgradient zeigte, war ein solcher 2011/12 nicht mehr feststellbar. Zurückzuführen ist dies vor allem auf eine überdurchschnittlich stark gesunkene Inanspruchnahme in den höheren Altersgruppen, die angesichts der hier deutlich häufigeren Beschwerden [27] sowie mit Blick auf das erklärte Ziel, ältere Beschäftigte länger im Erwerbsleben zu halten [28], nicht unproblematisch erscheint.

Bemerkenswert sind unsere geschlechts- und altersbezogenen Ergebnisse nicht zuletzt deshalb, weil die Präventionsleistungen der Krankenkassen gem. § 20 SGB V (Kurs- und Beratungsangebote nach dem sog. „,individuellen Ansatz“) durch Frauen und Versicherte im Alter von 40-60 Jahren regelmäßig stärker in Anspruch genommen werden als durch Männer bzw. junge Versicherte [5]. Dieses Muster scheint den von uns analysierten Daten zufolge bei Gesundheitsförderungsmaßnahmen im betrieblichen Kontext nicht - oder nur in stark abgeschwächter Form - zu existieren. Dies könnte zum Teil durch den Umstand erklärbar sein, dass die Angebotsgestaltung im Bereich der BGF insgesamt stärker auf die männlichen Beschäftigten ausgerichtet ist [4].

Dass der Grad der Beteiligung an BGF-Maßnahmen zwischen Arbeitern, Angestellten und Beamten nicht substantiell differiert, wurde in der vorliegenden Untersuchung erstmals auf repräsentativer Basis empirisch belegt. Ein eventueller Einfluss des sozialen Status der Beschäftigten auf die Inanspruchnahme lässt sich demnach nicht (mehr) an den genannten Kategorien festmachen. Auch aufgrund der im Beobachtungszeitraum vollzogenen tariflichen und sozialversicherungsrechtlichen Gleichstellung von Arbeitern und Angestellten sollten Zusammenhänge zwischen dem sozialen Status und der Beteiligung an BGF zukünftig besser anhand anderer Statusmerkmale (etwa: Stamm- vs. Randbelegschaft oder Niedrig- vs. Hochqualifizierte) untersucht werden.

Zwischen der BGF-Teilnahmequote und der Betriebsgröße besteht nach unseren Analysen ein inverser Zusammenhang. Dieser Befund deckt sich mit den Ergebnissen von Bödeker \& Hüsing, die einen derartigen Zusammenhang bei allen von ihnen unterschiedenen Arten von BGF-Maßnahmen fanden [12]. Die deutlich erhöhte Teilnahmequote in Kleinbetrieben mag nicht zuletzt daher rühren, dass deren „Kleinheit“ die direkte persönliche Ansprache und Information der Beschäftigten sowie die interkollegiale Motivation erleichtert. Andererseits kann hier aber auch rascher ein Konformitätsdruck entstehen, der es dem BGF-Angebot eher abgeneigten Beschäftigten relativ schwer macht, sich gegen eine Teilnahme zu entscheiden. Schließlich dürfte es in größeren Betrieben deutlich häufiger als in Kleinbetrieben vorkommen, dass BGF-Maßnahmen nur für ausgewählte Arbeitsbereiche (z.B. solche mit hohem Belastungsniveau oder auffälligem Krankenstand) gedacht sind und somit auch nur einem Teil der Belegschaft offenstehen.

\section{Schlussfolgerungen \\ $\nabla$}

Die Ergebnisse sprechen für eine im betrachteten Zeitraum moderat gestiegene Verbreitung von BGF in Deutschlands Betrieben, und zwar in allen Wirtschaftsbereichen und Betriebsgrößenklassen. Ungeachtet dieser insgesamt positiven Entwicklung verweisen die Ergebnisse auf einen nach wie vor erheblichen Beratungs- und Unterstützungsbedarf insbesondere der kleinen Betriebe. Genau zu beobachten ist auch, ob der für 2006-2012 festgestellte leichte Rückgang der BGF-Teilnahmequote insbesondere bei den älteren Beschäftigten sich zu einem anhaltenden Trend verfestigt, dem mit geeigneten Maßnahmen entgegengewirkt werden müsste. Generell verdient die Frage, ob mit BGF-Maßnahmen auch diejenigen Beschäftigten erreicht werden, die ihrer am meisten bedürfen, eine größere Aufmerksamkeit. Surveyergebnisse aus Dänemark deuten darauf hin, dass eine solche „Passung“ nicht durchgängig gegeben ist [29]. Anhand der BIBB/BAuA-Daten könnte dies - durch Analyse des Zusammenhangs von Belastungs- und Beschwerdeangaben einerseits und Angaben zur BGF andererseits - auch für Deutschland überprüft werden.

Für eine fundiertere Bewertung des Umsetzungsstands der BGF in Deutschland braucht es über die hier vorgestellten empirischen Informationen hinausgehende repräsentative Daten zur Struktur und Qualität der betrieblichen BGF-Praxis. Entsprechende Erhebungen und Analysen sollten sich jedoch nicht darauf beschränken, die Verbreitung und Nutzung verschiedener Einzelelemente von BGF (AU-Datenauswertungen, Befragungen, Gesundheitschecks, Bewegungsangebote, Stressbewältigung, Gesundheitszirkel usw.) darzustellen. Darzustellen wäre vielmehr auch, welche Konfigurationen von BGF-Maßnahmen (einschließlich solcher, die verhältnispräventiv ausgerichtet sind) die Betriebe umsetzen, um auf dieser Basis zumindest näherungsweise qualitative Niveaus betrieblicher Gesundheitsförderung und deren Verbreitung beschreiben zu können. Dies wäre ein wünschenswerter Beitrag zu einer differenzierteren Bewertung der BGF-Praxis in Deutschland.

Interessenkonflikt: Die Autoren geben an, dass kein Interessenkonflikt besteht.

\section{Literatur}

1 Faller G. Was ist eigentlich BGF? In: Faller G, Hrsg. Lehrbuch Betriebliche Gesundheitsförderung. 2. vollst. überarb. Aufl. Bern: Huber; 2012; 15-26

2 Luxemburger Deklaration zur betrieblichen Gesundheitsförderung in der Europäischen Union (i.d.F. vom Januar 2007) Im Internet www. dnbgf.de/fileadmin/texte/Downloads/uploads/dokumente/2011/Lu xemburger_Deklaration_09_11.pdf Stand: 22.01.2014

3 Reusch J, Lenhardt U, Kuhn J et al. Die Arbeitswelt von heute: Daten, Schwerpunkte, Trends. In: Schröder L, Urban H-J, Hrsg. Gute Arbeit: Profile prekärer Arbeit - Arbeitspolitik von unten. Ausgabe 2014. Frankfurt a.M.: Bund-Verlag; 2014; 349-384 
4 Medizinischer Dienst des Spitzenverbandes Bund der Krankenkassen e.V. (MDS). Präventionsbericht 2012. Leistungen der gesetzlichen Krankenversicherung: Primärprävention und betriebliche Gesundheitsförderung. Berichtsjahr 2011. Essen: MDS; 2012

5 Medizinischer Dienst des Spitzenverbandes Bund der Krankenkassen e.V. (MDS). Präventionsbericht 2013. Leistungen der gesetzlichen Krankenversicherung: Primärprävention und betriebliche Gesundheitsförderung. Berichtsjahr 2012. Essen: MDS; 2013

6 Bell F, Knoll A, Wegner B et al. Zwischen Gesundheitsförderung und Arbeitsschutz: Die Aktivitäten der Unfallversicherungsträger im Bereich „Gesundheit im Betrieb“. DGUV Forum 2010; 2: 26-31

7 Ulmer J, Groeben F. Work place health promotion. A longitudinal study in companies placed in Hessen and Thueringen. Journal of Public Health 2005; 13: 144-152

8 Gröben F, Wenninger S. Betriebliche Gesundheitsförderung im öffentlichen Dienst. Ergebnisse einer Wiederholungsbefragung von Führungskräften in Hessen und Thüringen. Prävention und Gesundheitsförderung 2006; 1: 94-98

9 Zelfel RC, Alles T, Weber A. Gesundheitsmanagement in kleinen und mittleren Unternehmen - Ergebnisse einer repräsentativen Unternehmensbefragung. Das Gesundheitswesen 2011; 73: 515-519

10 Landesinstitut für Gesundheit und Arbeit des Landes Nordrhein-Westfalen (LIGA NRW). Gesunde Arbeit NRW 2009. Belastung - Auswirkung - Gestaltung - Bewältigung. LIGA. Praxis 3. Düsseldorf: LIGA. NRW; 2009

11 Bechmann S, Jäckle R, Lück P et al. Motive und Hemmnisse für Betriebliches Gesundheitsmanagement (BGM). IGA-Report 20. Berlin, Essen: Initiative Gesundheit und Arbeit; 2010

12 Bödeker W, Hüsing T. IGA-Barometer 2. Welle. Einschätzungen der Erwerbsbevölkerung zum Stellenwert der Arbeit, zur Verbreitung und Akzeptanz von betrieblicher Prävention und zur krankheitsbedingten Beeinträchtigung der Arbeit - 2007. IGA-Report 12. Essen et al.: Initiative Gesundheit und Arbeit; 2008

13 Zok K. Stellenwert und Nutzen betrieblicher Gesundheitsförderung aus Sicht der Arbeitnehmer. In: Badura B, Schröder H, Vetter C, Hrsg. Fehlzeiten-Report 2008. Betriebliches Gesundheitsmanagement: Kosten und Nutzen. Heidelberg: Springer; 2009; 85-100

14 Hollederer A. Betriebliche Gesundheitsförderung in Deutschland - Ergebnisse des IAB-Betriebspanels 2002 und 2004. Das Gesundheitswesen 2007; 69: 63-76

15 Beck D, Schnabel P-E. Verbreitung und Inanspruchnahme von Maßnahmen zur Gesundheitsförderung in Betrieben in Deutschland. Das Gesundheitswesen 2010; 72: 222-227

16 Institut DGB-Index Gute Arbeit. Arbeitsfähig bis zur Rente? DGB-Index Gute Arbeit - Der Report. Ergebnisse der Repräsentativumfrage 2012 zur Ermittlung des DGB-Index Gute Arbeit. Berlin: Institut DGB-Index Gute Arbeit; 2013

17 TNS Infratest Sozialforschung. BIBB/BAuA-Erwerbstätigenbefragung 2011/2012. Feldbericht. München: TNS Infratest; 2012
18 TNS Infratest Sozialforschung. BIBB/BAuA-Erwerbstätigenbefragung 2011/2012. Strukturkontrolle, Steuerung und Gewichtung der Stichprobe. München: TNS Infratest; 2012

19 Nöllenheidt C, Wittig P, Brenscheidt S. Grundauswertung der BIBB/ BAuA-Erwerbstätigenbefragung 2012. Vergleich zur Grundauswertung 2006. Dortmund, Berlin, Dresden: Bundesanstalt für Arbeitsschutz und Arbeitsmedizin; 2014

20 TNS Infratest Sozialforschung. Repräsentative Befragung im Rahmen der Dachevaluation der Gemeinsamen Deutschen Arbeitsschutzstrategie (GDA).Tabellarische Grundauswertung, Tabellenband 1c: Gesamtergebnisse Arbeitnehmerbefragung, beschäftigtenproportional gewichtet. München: TNS Infratest; 2013

21 Kistler E, Trischler F. Repräsentative Analysen auf Basis des IAB-Betriebspanels 2012. Bericht an das Bayerische Staatsministerium für Arbeit und Sozialordnung, Familie und Frauen und die Regionaldirektion Bayern der Bundesagentur für Arbeit. Stadtbergen: Internationales Institut für Empirische Sozialökonomie (INIFES); 2013

22 Kuhn K. Der Betrieb als gesundheitsförderndes Setting: Historische Entwicklung der Betrieblichen Gesundheitsförderung. In: Faller G, Hrsg. Lehrbuch Betriebliche Gesundheitsförderung. 2. vollst. überarb. Aufl. Bern: Huber; 2012; 27-38

23 Kliche T, Kröger G, Meister R. Die Implementation betrieblicher Gesundheitsförderung in Deutschland: Stand, Hürden und Strategien ein Überblick. In: Kirch W, Middeke M, Rychlik R, Hrsg. Aspekte der Prävention. Stuttgart: Thieme; 2009; 224-235

24 Beck D. Zeitgemäße Gesundheitspolitik in Kleinst- und Kleinbetrieben. Hemmende und fördernde Bedingungen. Berlin: Edition Sigma; 2011

25 Ellguth $P$, Kohaut $S$. Tarifbindung und betriebliche Interessenvertretung. Ergebnisse aus dem IAB-Betriebspanel 2012. WSI-Mitteilungen 2013; 66: 281-288

26 Ellguth $P$, Kohaut S. Der Staat als Arbeitgeber: Wie unterscheiden sich die Arbeitsbedingungen zwischen öffentlichem Sektor und der Privatwirtschaft? Industrielle Beziehungen 2011; 18: 11-38

27 Bundesministerium für Arbeit und Soziales, Bundesanstalt für Arbeitsschutz und Arbeitsmedizin. Sicherheit und Gesundheit bei der Arbeit 2012. Unfallverhütungsbericht Arbeit. Dortmund, Berlin, Dresden: Bundesanstalt für Arbeitsschutz und Arbeitsmedizin; 2013

28 Bundesministerium des Inneren. Jedes Alter zählt. Demografiestrategie der Bundesregierung (25.04.2012). Im Internet http:// www.bundesregierung.de/Webs/Breg/DE/Themen/Demografie strategie/Artikel/Anlagen/demografiestrategie-langfassung.pdf?_ blob $=$ publicationFile $\& V=2$ Stand: 15.01 .2014

29 Jørgensen $M B$, Villadsen E, Burr $H$ et al. Does workplace health promotion in Denmark reach relevant target groups? Health Promotion International 2013, doi:10.1093/heapro/dat041 